\title{
Core stability in adolescent swimmers with swimmer's shoulder syndrome
}

\author{
DOI: https://doi.org/10.5114/pq.2021.105751
}

\author{
Azza Mohammed Abdelmohsen', Salam Mohamed Elhafez', Bassam Ahmed Nabil ${ }^{2}$ \\ ${ }^{1}$ Biomechanics Department, Faculty of Physical Therapy, Cairo University, Giza, Egypt \\ ${ }^{2}$ Biomechanics Department, Faculty of Physical Therapy, October 6 University, Giza, Egypt
}

\section{Abstract}

Introduction. Swimmer's shoulder syndrome (SSS) is the most common overuse injury affecting swimmers and surfers. Core stability is crucial to prevent SSS. The study investigated the effect of isokinetic core strength of trunk flexors and extensors and core endurance on shoulder stability in adolescent swimmers with SSS.

Methods. The study involved 30 swimmers, assigned to 2 equal groups. The experimental group (A) suffered from SSS; the control group (B) included healthy swimmers with no history of shoulder pain or instability. The mean values of age, body mass, and height were $12.86 \pm 1.59$ years, $41.73 \pm 3.99 \mathrm{~kg}$, and $142.0 \pm 3.96 \mathrm{~cm}$, respectively, in group A and $13.2 \pm 1.56$ years, $42.66 \pm 3.9 \mathrm{~kg}$, and $142.26 \pm 4.39 \mathrm{~cm}$, respectively, in group B. Isokinetic peak torque (PT) for trunk flexors and extensors was measured with a Biodex isokinetic dynamometer. Four functional tests assessed core endurance: side bridge endurance test, static back endurance test, ball bridge test, and unilateral bridge test.

Results. There was a statistically significant between-group difference in mean PT of trunk extension at both angular velocities: $60 \%, 180 \%$. No significant differences were detected in mean PT of trunk flexion. All functional core stability tests revealed a greater endurance time in group $B$.

Conclusions. Weak core inhibits shoulder muscles, which is a major risk factor for shoulder instability and SSS. It is crucial to incorporate core stability training into the rehabilitation plan to provide proximal stability for obtaining safe and proper distal mobility across the shoulder joint.

Key words: shoulder dislocation, shoulder pain, central core, muscle strength

\section{Introduction}

Shoulder pain is a major problem that leads to muscle inhibition. This, in turn, affects muscle strength and joint stability, augmenting the problem and adding another risk factor to shoulder injuries [1]. O'Donnell et al. [2] reported that swimming was a sport applying a remarkable overload on shoulders. This is frequently accompanied by an undesirable increase in joint laxity. Numerous swimmers exhibited signs of scapular dyskinesia through the course of a 100-minute swimming session [3]. Elite-level competitive swimmers accomplish between 1500 and 4000 stroke cycles per day, or about 1,000,000 stroke cycles per year [4]. In the same context, Oyama [5] demonstrated that swimmers typically performed very high repetitions of strokes compared with baseball throwers and tennis players. Therefore, swimming requires the shoulder to perform work over a greater period of time and thus a greater number of repetitions, and this needs an incorporated action of a group of muscles that act in a much more synergetic way to obtain the required force with minimal joint loads. These muscles are the shoulder girdle musculature and core stabilizers.

Swimmer's shoulder syndrome (SSS) is the general term for an overuse injury in swimming athletes in which they suffer from shoulder pain and instability [6, 7]. Shoulder pain is the most common musculoskeletal complaint in swimmers and is typically classified as a chronic injury due to repetitive overuse [8-10]. The National Collegiate Athletic Association revealed through a 5-year survey that the whole elite swimmer injury rates were 4 injuries per 1000 hours of training for men and 3.78 injuries per 1000 hours training for women [11]. The cause of shoulder pain in swimmers is considered multifactorial and may involve many different aetiologies related to poor biomechanics. These causative factors include the subacromial impingement syndrome, overuse and subsequent muscle fatigue, scapular dyskinesia, laxity and instability, labral damage, os acromiale, and suprascapular neuropathy $[8,12]$. Microtears or stretching of the glenohumeral ligaments aggravate the static instability. As soon as these athletes experience fatigue, their rotator cuff muscles become unable to stabilize the humerus alone. This increases the translation of the humeral head, mainly upward or superior-posterior, overloading the rotator cuff tendon. Shoulder abduction and rotation with excessive humeral head translation lead to the contact of the posterior supraspinatus or anterior infraspinatus against the posterior glenoid rim and labrum. This disorder is described as internal impingement [13]. Scapular protraction is a common sign of scapular dyskinesia in swimmers $[3,14]$.

The core of the body includes both passive and active structures: the passive structures of the thoracolumbar spine and pelvis and the active contributions of the trunk musculature. In other words, spine stability depends not only on muscular strength, but also on the proper sensory input that alerts the central nervous system about interaction between the body and the environment, providing constant feedback and allowing refinement of movement. The core is anatomically defined as a muscular box. The boundaries of this box are designed as follows: the abdominal muscles in the front, paraspinal and gluteal muscles in the back, the diaphragm as the roof, and the pelvic floor and hip girdle musculature as the bottom [15]. Within this box, there are 29 pairs of muscles

Correspondence address: Azza Mohammed Abdelmohsen, Biomechanics Department, Faculty of Physical Therapy, Cairo University, 7 Ahmed Ezzayyat Street, Bein Essarayat, Giza, Egypt, e-mail: dr_azzamohammed@yahoo.com

Received: 22.04.2020

Accepted: 19.06 .2020

Citation: Abdelmohsen AM, Elhafez SM, Nabil BA. Core stability in adolescent swimmers with swimmer's shoulder syndrome. Physiother Quart. 2021;29(4):33-41; doi: https://doi.org/10.5114/pq.2021.105751. 
that stabilize and support the spine, pelvis, and kinetic chain during functional movements. The spine cannot be mechanically stable without these muscles as the compressive forces placed on it are at least $90 \mathrm{~N}$. Once the core muscular system works properly, the consequence is appropriate force distribution and maximum force $[15,16]$. This is not only the core benefit; also, it decreases the compressive, translational, and shearing forces at the joints of the kinetic chain to the minimal value, reducing the loads on the upper and lower extremities. In overhead activities, a strong core minimizes loads on the shoulder joint, providing a base for safe shoulder movement. In addition, the diaphragm muscle (the roof of the core) delivers good respiration that ensures good posture and efficient shoulder movement. Core stability is perceived as being a key for well-organized biomechanical function to augment force generation and diminish joint loads in all kinds of activities, ranging from running to swimming [16]. Kibler et al. [14] defined core stability as the capability to control the position and motion of the trunk over the pelvis to enhance the peak production, transfer, and control of force and motion to the distal segment in incorporated athletic activities.

A strong core zone qualifies an athlete to fully transfer ground reaction forces from the lower extremities to the torso, and eventually to the upper extremities. Better core strength and endurance improve sports performance. The core serves as the centre of the functional kinetic chain. The core is seen as a muscular corset that works as a unit to stabilize the body, in particular the spine, both with and without limb movement. In the alternative medicine world, the core has been referred to as the 'powerhouse,' the foundation or engine of all limb movement $[17,18]$. An efficient core ensures good flexibility of trunk flexors and extensors, which makes it easy for the human movement system to sustain favourable force couple relationships throughout the lumbo-pelvic-hip complex (LPHC) $[19,20]$. This neuromuscular capability of the core is habitually known as core stability, but it is more accurately described as LPHC stability, consisting of local or intersegmental stability (local stabilization system), global stability (global stabilization system), and global mobility [21, 22]. This interdependent system needs to be properly trained to empower it to function professionally throughout dynamic activities. High-level sports performance requires functional strength, power, neuromuscular control, and muscular endurance in definite muscles.

SSS should perfectly be diagnosed, and concentrated functional rehabilitation of the shoulder girdle and core muscles should be started as early as possible to regain muscle balance [23]. Isokinetic testing is a critical part of shoulder injury evaluation. Research has established its value in obtaining clinically relevant information concerning muscular performance [24]. Shoulder injury is first managed through core stabilization and then by scapular stabilization [25, 26].

Up to the authors' knowledge, there are few studies that assessed the isokinetic and functional strength of core muscles in adolescent swimmers. Adolescence time is very important for athletes, especially swimmers, to be assessed for injury risk. This, in turn, increases swimmers' awareness of and concern for prevention of risk factors that predispose to injuries. Therefore, the purpose of this study was to investigate the effect of trunk muscle weakness on shoulder stability. This may help predict the diagnosis, prognosis, and treatment of SSS. Moreover, it may help protect swimmers from possible risk factors for shoulder pathology in order to enable them to elevate their level of performance and eliminate shoulder pain by incorporating core stability training into the rehabilitation program of such cases.

\section{Subjects and methods}

\section{Participants}

Thirty adolescent competitive swimmers of both sexes (16 males and 14 females) volunteered to participate in this study. They were college students at the preparatory stage of learning. They had 2-5 years of swimming practice and showed a good swimming style, passing the $3^{\text {rd }}$ Star test of the Egyptian Swimming Federation. They swam 3000-7000 m per day and performed 8-14 hours/week of swimming sessions and 3-6 hours/week of dryland sessions. They were assigned into 2 equal groups of 15 . There were no potential differences between the 2 groups. The experimental group (A) were referred by a physician and had a diagnosis of SSS. However, at the time of testing, they were pain-free to avoid muscle inhibition due to pain. The control group (B) were healthy swimmers with no history of shoulder pain or instability. The mean values of age, body mass, and height were $12.86 \pm 1.59$ years, $41.73 \pm 3.99 \mathrm{~kg}$, and $142.0 \pm 3.96 \mathrm{~cm}$, respectively, in group $A$ and $13.2 \pm 1.56$ years, $42.66 \pm 3.9 \mathrm{~kg}$, and $142.26 \pm 4.39 \mathrm{~cm}$, respectively, in group B. Normality tests (Shapiro-Wilk) were applied for these variables for data exploration to exclude outliers before conducting the suitable statistical analysis. Once the normality tests revealed normal data distribution for these variables, parametric statistical analysis using an independent $t$-test was conducted. The results demonstrated no significant differences in the mean values of the participants' demographic data, including age, weight, and height, between the 2 groups, as shown in Table 1.

Table 1. Participants' demographic data

\begin{tabular}{|c|c|c|c|}
\hline Characteristics & $\begin{array}{c}\text { Experimental } \\
\text { group } \\
(n=15: \\
8 \text { males, } \\
7 \text { females) } \\
\text { (mean } \pm S D \text { ) }\end{array}$ & $\begin{array}{c}\text { Control } \\
\text { group } \\
(n=15: \\
8 \text { males, } \\
7 \text { females) } \\
\text { (mean } \pm S D)\end{array}$ & $\begin{array}{l}\text { Experimental } \\
\text { group vs. } \\
\text { control group } \\
\text { (p) }\end{array}$ \\
\hline Age (years) & $12.86 \pm 1.59$ & $13.2 \pm 1.56$ & 0.52 \\
\hline Weight (kg) & $41.73 \pm 3.99$ & $42.66 \pm 3.9$ & 0.56 \\
\hline Height (cm) & $142.0 \pm 3.96$ & $142.26 \pm 4.39$ & 0.86 \\
\hline
\end{tabular}

\section{Eligibility criteria}

Swimmers were included in group A if they met the following criteria: (1) Their problem started 6-12 months earlier and they were diagnosed by the referring physician as having SSS with symptoms of pain, muscle imbalance, tendinitis, impingement, bursitis, and instability. During the time of testing, they were pain-free (asymptomatic) to avoid muscle inhibition. (2) The swimmer was diagnosed with appropriate imaging techniques, including plain films (X-ray including an outlet view to fully visualize the acromion) and/or magnetic resonance imaging. (3) They had unilateral affection (dominant side).

Participants were excluded from the study if they were characterized by any of the following: (1) Traumatic injury or surgery related to shoulder and trunk. (2) Breast type swimming stroke. (3) Severe shoulder laxity exhibiting signs of ligamentous hyperlaxity elsewhere in the body, such as hyperextension of the elbow, knees, fingers, or hand. (4) Other overhead sport practice. (5) Rheumatoid arthritis or other inflammatory joint disease. (6) Previous surgical operation or injury in the neck, trunk, or upper or lower limbs. 


\section{Core stability assessment}

Each swimmer was assessed 24-48 hours after the last training (swimming or dryland session). This was done to minimize the difference of training load impact either over training or unloading of muscles.

Measuring trunk muscle strength via a Biodex System 3 Pro isokinetic dynamometer

A Biodex multi-joint testing and rehabilitation system (Biodex Medical Systems, Shirley, NY, USA) was used in this

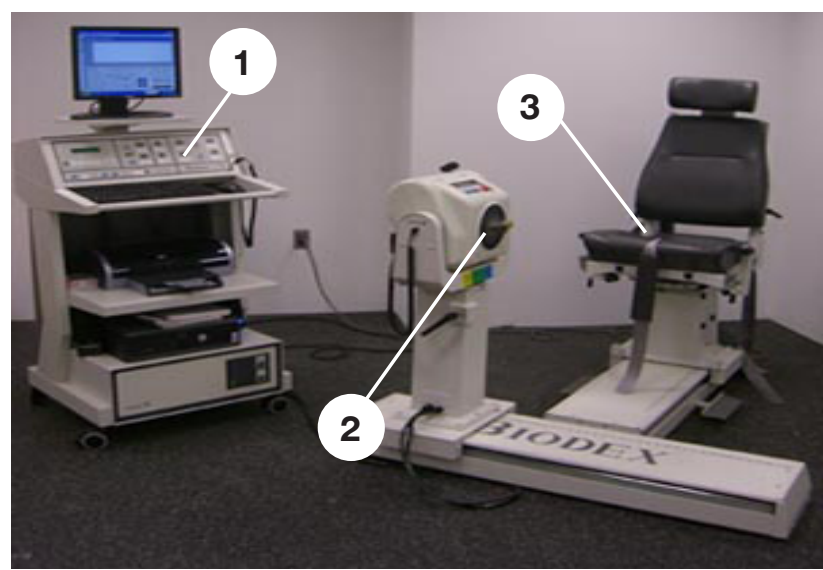

Figure 1. Biodex isokinetic multi-joint system: 1 - control unit, 2 - head assembly, 3 - positioning chair

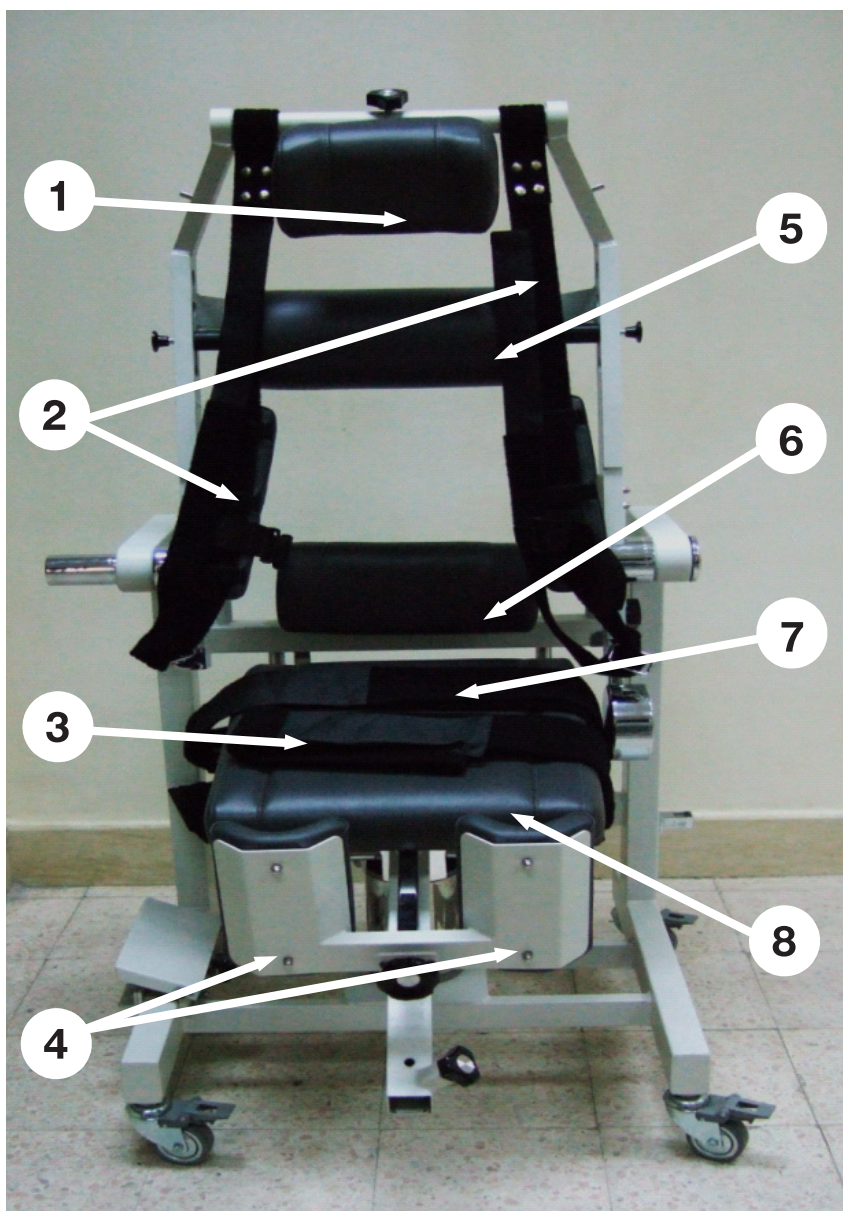

Figure 2. Trunk attachment with its components:

1 - adjustable head seat 5 -thorax padded roller bar

2 - chest straps $\quad 6$-lumbar support pad

3 - thigh strap

7 - pelvic strap

4 - anterior leg pads study to measure the isokinetic trunk flexion and extension peak torque (PT), which expresses muscle strength. The Biodex isokinetic dynamometer has been widely used in research, clinical setting, and rehabilitation to objectively assess factors of muscles performance that would otherwise be difficult to obtain by using the manual testing technique [27]. The isokinetic dynamometer maintains a constant velocity while giving an accommodating resistance throughout a joint range of motion when measuring internal PT [28]. Performance measures were automatically recorded by the system's custom software at the different angular velocities [29]. The Biodex isokinetic dynamometer used in the current study consists of a dynamometer head, positioning chair, and control unit. The main parts of the device are illustrated in Figures 1 and 2. Moreover, the Biodex software compensates for the effects of gravity as part of the setup with the subjects positioned appropriately [30].

The procedures of the isokinetic trunk flexion and extension tests were as follows (Figures 3-5):

- The trunk seated compressed protocol was used, isolating trunk movement, with no pelvic or hip muscles sharing. The participant was allowed to sit on the adjustable seat of the Biodex isokinetic dynamometer system in the optimal resting position that allowed a greater range of motion both in flexion and extension and hence was the preferred testing position [31, 32].

- The dynamometer head was coupled with chair attachment for testing the trunk.

- The pelvic strap was then applied and positioned as far as possible to press firmly, but comfortably, against the superior aspect of the proximal thighs. Two curved anterior leg pads were secured to adjust the knee block position. In addition, a lumbar support pad was located against the lower lumbar spine. Therefore, the pelvis was stabilized to minimize any contribution from the hip muscles [31]. Both thighs were then stabilized by another strap and the feet were held in place without being in contact with the floor.

- The participant sat erect with the head stabilized neutrally against an adjustable head seat. The 2 anterior force application straps were aligned vertically and then connected to another horizontal strap, which was aligned with the second intercostal cartilage on the anterior chest wall when measuring the flexion torque. The posterior force application padded roller bar was placed on the posterior trunk just distal to the spine of the scapula when measuring the extension torque.

- The range of motion was set from $70^{\circ}$ lumbar extension (obligatorily limited by a metal bar implemented behind the lumbar region) to $20^{\circ}$ trunk flexion and limited by contacting the arm attachment with the subject's thigh.

- Isokinetic testing was performed at 2 angular velocities: $60 \%$ s, then $180 \%$ s. Concentric mode of contraction was selected in the control panel of the computer unit.

- To prevent any jerky or unintended arm movement, the participant was instructed to rest the crossed forearms on the anterior chest wall. In addition, the participant was requested to maintain a neutral head position throughout the testing procedure to avoid any contribution from the neck muscles [33].

- The subject was instructed to hold the attachment firmly and perform 10 maximal concentric contractions at an angular velocity of $60 \%$ s.

- There was a 60-second break between the trials (after each 10 repetitions) and $\mathrm{PT}$ was recorded.

- The same was repeated at an angular velocity of $180 \%$ s.

- After finishing all trials, each participant was given 5 minutes for cooling down (in the form of respiratory exercise) to avoid any post-test complications. 


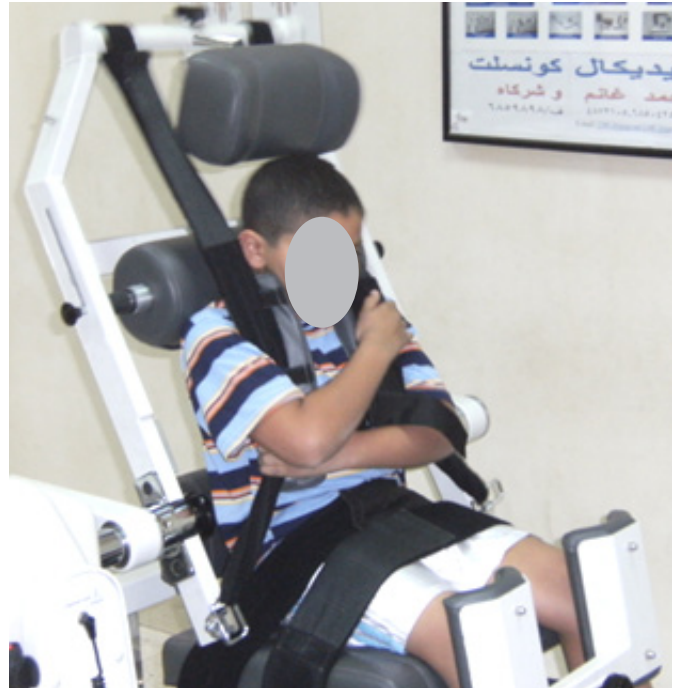

Figure 3. Starting position of isokinetic trunk flexion and extension tests

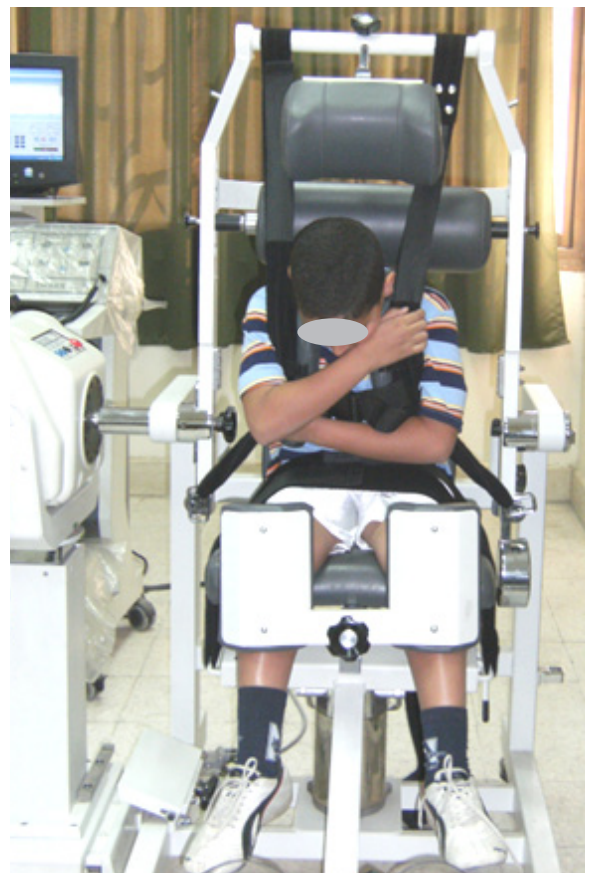

Figure 4. Isokinetic trunk flexion test

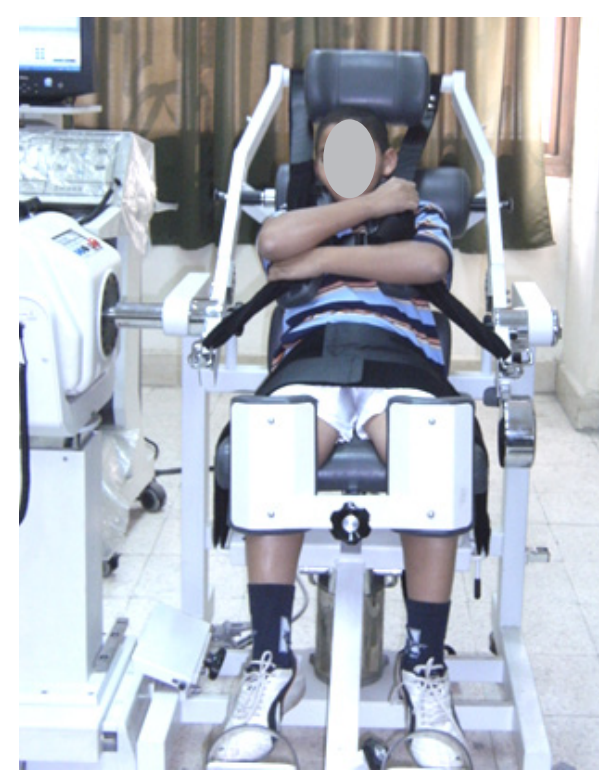

Figure 5. Isokinetic trunk extension test
Core endurance functional tests

Four tests (in non-weight-bearing positions) [22] were performed in a similar order to ensure the same successive loading. The tests were: (1) side bridge endurance test (quadratus lumborum endurance stretch), (2) static back endurance test or prone extension endurance test (paraspinal endurance strength), (3) ball bridge test, and (4) unilateral bridge test. In each test, the participant was instructed to maintain the position as long as they could to calculate core endurance time, and the test was repeated 3 times. Then, the average time was calculated in seconds.

Side bridge endurance test. The participant assumed a side-lying position on the floor while the feet and the forearm were closest to the ground and the contralateral forearm was set across the chest. The legs were extended. The subject was instructed to lift the body up off the floor while supported on the dominant side and hold this position as long as possible while supporting the body weight. The test position is shown in Figure 6.

Static back endurance test (prone extension endurance test). The participant assumed a prone lying position on a flat bench, with the ankles anchored underneath a pad. The tip of the iliac crest was rested on the edge of the bench and the arms were folded across the chest. The participant was instructed to lift the body up off the floor and hold this position for as long as possible. They assumed a horizontal position that was secured by using a water scale put along the lower spine (lumbar, sacrum, and midway between the buttocks), as illustrated in Figure 7.

Ball bridge test. The participant assumed a supine lying position, with both heels rested on a medical ball; both arms were placed beside the body, with the palms contacting the ground. The subject was instructed to lift the body up off the floor and hold this position for as long as possible [34], as demonstrated in Figure 8.

Unilateral bridge test. The participant assumed a halfcrook lying position, with a knee flexed at $90^{\circ}$. The sole of foot was rested on the ground. The other leg was extended, with the heel contacting the ground. The subject supported them-

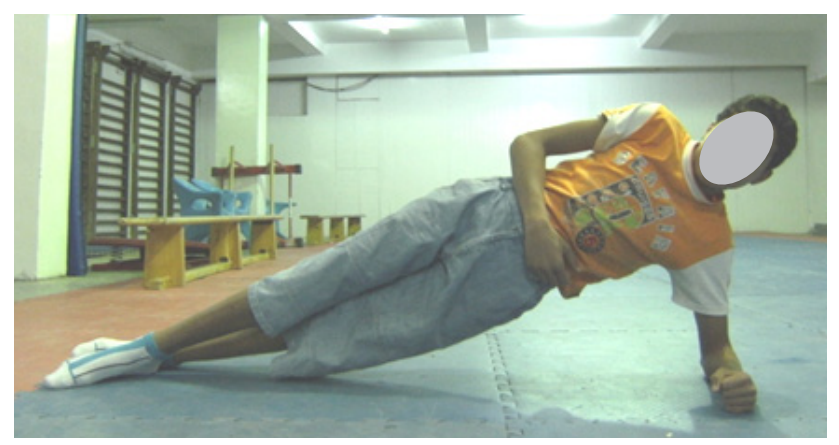

Figure 6. Side bridge endurance test

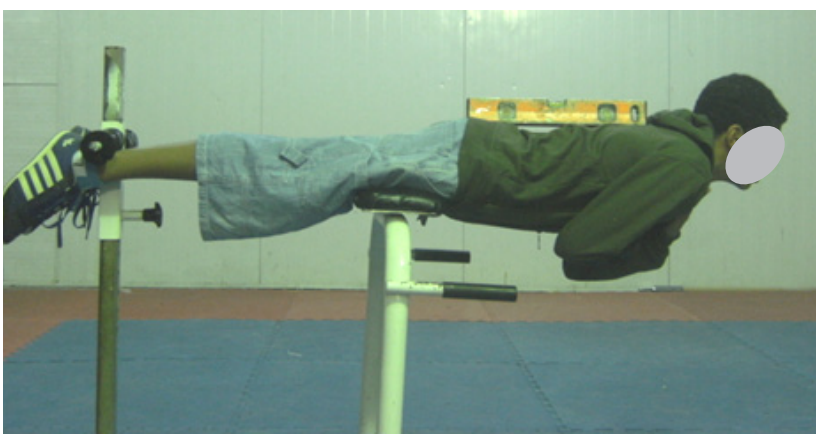

Figure 7. Position of static back endurance test 


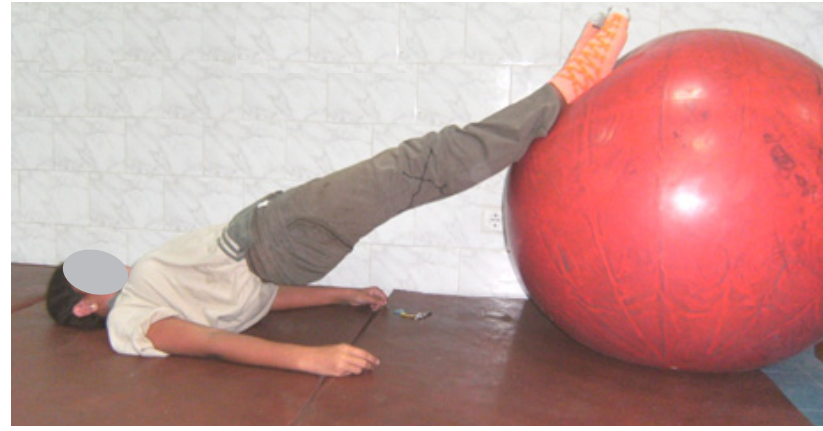

Figure 8. Ball bridge test

selves on the flexed leg, and lifted the body and the extended leg up off the floor, then held this position for as long as possible, while supporting the body weight and the extended leg [34], as shown in Figure 9. The stopwatch was started when the subject assumed the correct position and was stopped when the position was no longer maintained. The test was repeated for the contralateral leg. The average time for the right leg was added to that for the left leg and then divided by 2 to gain the total average time. Each participant had 5 minutes for cooling down in the form of respiratory exercise, gentile stretch of the entire body, and pendulum exercises for either upper or lower extremity to avoid any posttest complications.

\section{Statistical analysis}

First, data exploration was carried out to test the homogeneity of variances between the groups. Normality test results (Shapiro-Wilk) were insignificant $(p>0.05)$, revealing homogeneity, and frequency distribution curves showed normal skewness and kurtosis after excluding outliers. Then, oneway between-subject design multivariate analysis of variance (MANOVA) was used to assess the isokinetic PT for trunk flexors and extensors in addition to functional core stability between group $A$ and group $B$. The study included one independent variable: the tested group (between-subject factor) with 2 levels, group A and group B. The 6 dependent variables were the isokinetic PT for trunk flexors and trunk extensors, as well as the results of the 4 functional core stability tests, including side bridge endurance test, static back endurance test, ball bridge test, and unilateral bridge test. The statistical analysis was performed by using the Statistical Package for the Social Sciences (SPSS), version 20 for Windows. The level of significance for all statistical tests was set at $p<0.05$.

\section{Ethical approval}

The research related to human use has complied with all the relevant national regulations and institutional policies,

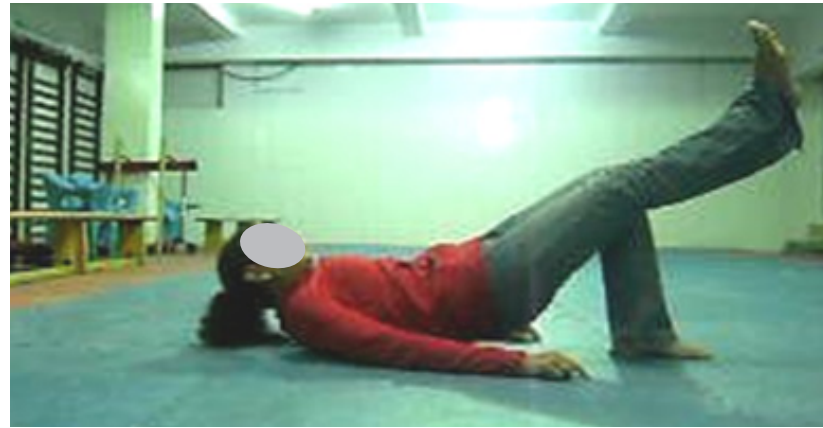

Figure 9. Unilateral bridge test

has followed the tenets of the Declaration of Helsinki, and has been approved by the Research Ethics Committee of Faculty of Physical Therapy, Cairo University.

\section{Informed consent}

Informed consent has been obtained from the parents of all individuals included in this study.

\section{Results}

\section{Isokinetic trunk flexion and extension peak torque}

There were no significant differences between group $A$ and group $B$ in the mean trunk flexion PT values at either angular velocity $(60 \%$ s or $180 \% / s)(p>0.05)$. In turn, group B showed a significant difference in trunk extension PT values compared with group $A$ at both angular velocities $(60 \%$ s and $180 \%$ s) $(p=0.001)$. These results are summarized in Table 2 and Figure 10.

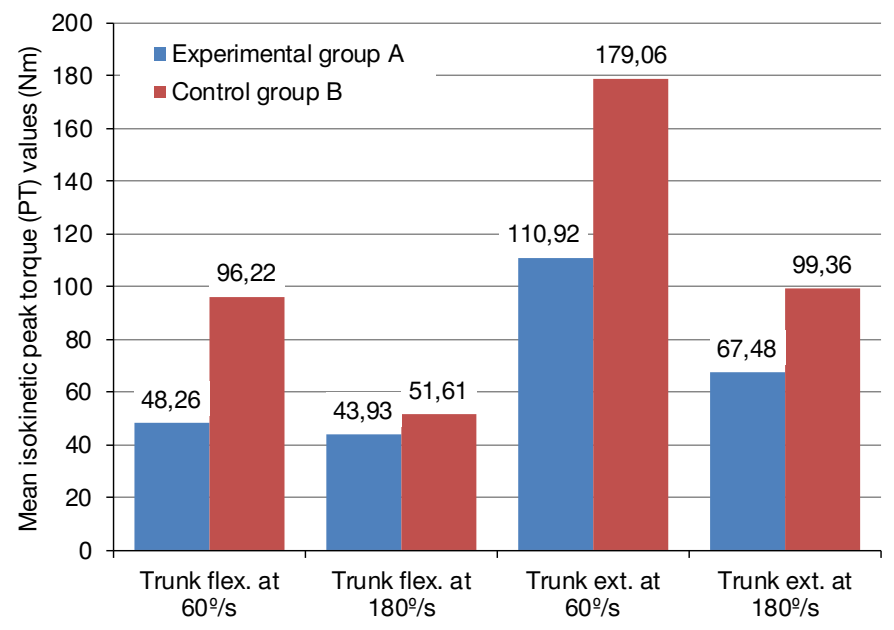

Figure 10. Mean isokinetic peak torque values of trunk flexion and extension measured at the angular velocities of $60 \%$ and $180 \%$ s for both groups

Table 2. Results of one-way MANOVA for peak torque values of trunk flexion and extension measured at the angular velocities of $60 \%$ s and $180 \%$ s for both groups

\begin{tabular}{|c|c|c|c|c|}
\hline \multirow{2}{*}{$\mathrm{PT}(\mathrm{N} \cdot \mathrm{m})$} & \multirow{2}{*}{$\begin{array}{l}\text { Experimental group }(\mathrm{A}) \\
(\text { mean } \pm S D)\end{array}$} & \multirow{2}{*}{$\begin{array}{l}\text { Control group (B) } \\
\quad(\text { mean } \pm S D)\end{array}$} & \multicolumn{2}{|c|}{ MANOVA test } \\
\hline & & & $F$ & $p$ \\
\hline Trunk flexion PT at $60 \% / s$ & $48.26 \pm 17.29$ & $96.22 \pm 19.26$ & 3.19 & 0.08 \\
\hline Trunk flexion PT at $180^{\circ} / \mathrm{s}$ & $43.93 \pm 11.53$ & $51.61 \pm 14.3$ & 2.62 & 0.11 \\
\hline Trunk extension PT at $60^{\circ} / \mathrm{s}$ & $110.92 \pm 31.43$ & $179.06 \pm 24.93$ & 43.26 & $0.001^{*}$ \\
\hline Trunk extension PT at $180^{\circ} / \mathrm{s}$ & $67.48 \pm 13.34$ & $99.36 \pm 31.92$ & 12.73 & $0.001^{*}$ \\
\hline
\end{tabular}

* significant at alpha level $<0.05$ 
Table 3. Results of one-way MANOVA for core stability functional tests

\begin{tabular}{|c|c|c|c|c|}
\hline \multirow{2}{*}{ Test results (s) } & \multirow{2}{*}{$\begin{array}{l}\text { Experimental group }(A) \\
\quad(\text { mean } \pm S D)\end{array}$} & \multirow{2}{*}{$\begin{array}{l}\text { Control group (B) } \\
\quad(\text { mean } \pm S D)\end{array}$} & \multicolumn{2}{|c|}{ MANOVA test } \\
\hline & & & $F$ & $p$ \\
\hline Side bridge endurance test & $38.4 \pm 4.82$ & $85.86 \pm 9.17$ & 314.31 & $0.001^{*}$ \\
\hline Static back endurance test & $59.53 \pm 11.91$ & $101.53 \pm 9.25$ & 116.21 & $0.001^{*}$ \\
\hline Ball bridge test & $151.73 \pm 28.73$ & $227.26 \pm 14.54$ & 82.49 & $0.001^{*}$ \\
\hline Unilateral bridge test & $98.96 \pm 20.67$ & $160.53 \pm 17.17$ & 80.48 & $0.001^{*}$ \\
\hline
\end{tabular}

${ }^{*}$ significant at alpha level $<0.05$

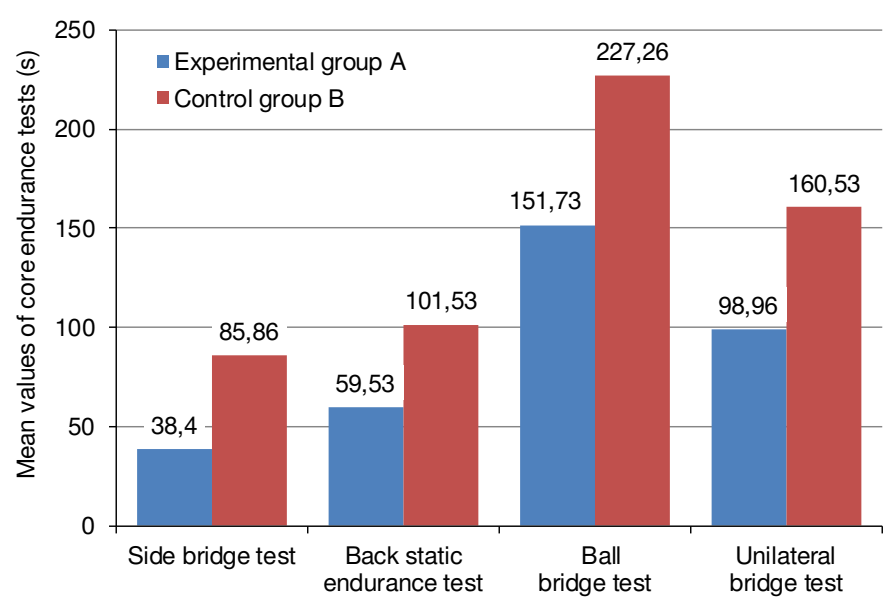

Figure 11. Mean values of core stability functional tests

\section{Core endurance functional tests}

Group B exhibited a significant difference in the endurance time compared with group $A$ in the 4 functional core stability tests: side bridge endurance test, static back endurance test, ball bridge test, and unilateral bridge test. The mean time values for the side bridge endurance test were $85.86 \pm 9.17 \mathrm{~s}$ and $38.4 \pm 4.82 \mathrm{~s}$ for group $\mathrm{B}$ and group $\mathrm{A}$, respectively $(p=0.001)$. The mean time values for the static back endurance test were $101.53 \pm 9.25 \mathrm{~s}$ and $59.53 \pm$ $11.91 \mathrm{~s}$ for group $\mathrm{B}$ and group $\mathrm{A}$, respectively $(p=0.001)$. In the ball bridge test, the achieved endurance times equalled $227.26 \pm 14.54 \mathrm{~s}$ in group $B$ and $151.73 \pm 28.73 \mathrm{~s}$ in group $A$ $(p=0.001)$. Similarly, the mean values of the unilateral bridge test were $160.53 \pm 17.17 \mathrm{~s}$ and $98.96 \pm 20.67 \mathrm{~s}$ for group $\mathrm{B}$ and group $A$, respectively $(p=0.001)$. All functional core stability scores are summarized in Table 3 and Figure 11.

\section{Discussion}

\section{Isokinetic peak torque of trunk flexion and extension}

This study revealed that the mean values of isokinetic trunk extension PT were significantly different in group B compared with group A, suffering from SSS, at both angular velocities: $60 \%$ and $180 \%$ s. This result confirms the relationship between weak core and shoulder instability. Trunk extensors have a very important role in swimming to allow efficient transfer of force from lower limbs to trunk and then to upper limbs, minimizing overload on the shoulder joint [35]. Within time, poor body positioning or weak back muscles can contribute to shoulder pain both during and after workouts. However, despite the greater value of trunk flexion PT in group B compared with group $A$, no statistically significant difference was obtained between the 2 groups at either angular velocity flexion PT value between the groups may be attributed to muscle imbalance between trunk flexors and extensors. Trunk extensors are more activated and overloaded in swimming. The mechanics of trunk extensors is needed to maintain horizontal body position (prone and supine) during swimming without stopping, thus enabling swimmers to glide straight forward through the water with less resistance. This, in turn, increases the load placed on trunk extensors during swimming. Trunk extensors are also necessary in the floating horizontal position as the centre of gravity becomes near to the heavier lower body, unlike the centre of buoyancy, which becomes near to the lighter upper body (lungs inflated with air). The force couple created by these 2 forces will rotate and drop the lower body (trunk and lower extremities). As for the majority of time, the workout in swimming training is performed with freestyle stroke (prone), the swimmer needs a high strength from trunk extensors to overcome the sinking force couple. This, in turn, produces imbalance between trunk flexors and extensors. This imbalance may: (a) affect the horizontal position of swimmers to be slightly stooped; (b) affect the 2 force couples that control anterior and posterior pelvic tilting (lumbopelvic rhythm), with subsequent lumbar curve changes (mainly a flattened curve or, rarely, hyperlordosis); (c) change the muscle length tension relationship. These changes can decrease the gliding of the swimmer through the water, which puts more resistance on the body, so the swimmer requires more internal forces for locomotion, mainly from the upper extremities [36].

As reported by Kibler et al. [14], the new approach to the rehabilitation of shoulder joint that focuses on movement patterns addresses glenohumeral motion through scapular control and trunk movement. Fritz et al. [37] demonstrated that the core acted through the thoracolumbar fascia (TLF), 'nature's back belt,' which is responsible for a cooperated trunk stability, playing a significant role in shoulder torque production [38]. This is consistent with the proximal-to-distal kinetic link model of biomechanics and applies recent theories of motor control and closed kinetic chain exercise [39]. The core (LPHC) is stabilized during functional movement by 2 principal mechanisms: the thoracolumbar stabilization mechanism and the intra-abdominal pressure stabilization mechanism [22]. The TLF stabilization mechanism is accomplished through a fascial system of noncontractile tissue that plays an important role in the functional stability of the core and is divided into the posterior, anterior, and middle layers. Although TLF is noncontractile, it can be involved dynamically because of the contractile tissue that attaches to it. The muscles that attach to TLF include the deep erector spinae, multifidus, transverse abdominis, internal oblique, gluteus maximus, latissimus dorsi, and quadratus lumborum [40]. The transverse abdominis and the internal oblique muscles are chiefly important for stabilization. They attach to the middle layer of TLF via the lateral raphe, and the activation of both generates a traction and tension force on TLF, which 
augments the regional intersegmental stability in the core, reducing the translational and rotational stress. Basically, TLF serves as part of a 'hoop' around the trunk that provides a connection between the lower limb and the upper limb [15]. The second stabilization mechanism involves the intra-abdominal pressure mechanism. Increased intra-abdominal pressure decreases the compressive forces in the core. The abdominal muscles contract against the viscera, pushing the viscera superiorly into the diaphragm and inferiorly into the pelvic floor, and providing the intersegmental stabilization to the core (LPHC) [41].

\section{Core endurance functional tests}

This study indicated that there was a significant difference in the scores of all functional core endurance tests (the side bridge endurance test, static back endurance test, ball bridge test, and unilateral bridge test) between group $B$ and group A. This means that the efficiency of core stabilizers was smaller in swimmers with SSS than in healthy swimmers. This, in turn, affected the swimmers' performance during swimming and during testing. It also explains the strong relationship between shoulder pain and low core stability efficiency. Each of them can lead to the other, creating a vicious circle. In swimming, the fluid medium (water) needs high mechanical coordination between the core stability of LPHC and the speed mobility of upper and lower extremities. The forces start from the core region and then are directed to the more distal regions. Moreover, core strength is required to overcome the dipping force couple created by the centre of gravity and centre of buoyancy, as discussed before. Core has an important role in absorbing high load, protecting other delicate parts, like the shoulder, and is considered the central station that is necessary to develop force production and reduce joint loads in all types of activities. Additionally, core stability may be required to control the position and motion of the trunk to allow optimum production, transfer, and synchronization of force and motion to the distal limbs and, finally, to hands and feet to integrate swimming activities. Thus, weak core may participate in developing SSS.

These results are in line with those achieved by Johnson et al. [42], who recognized the need to address the legs and trunk as contributors to shoulder function and for general conditioning. They used electromyography to prove that the rapid right shoulder forward reaching produced a consistent pattern of activation and deactivation of leg and trunk musculature (core stabilizers) before the activation of the anterior deltoid. They reported that in the kinetic chain, the legs and trunk were integrated into most of the shoulder exercises from the onset and were activated before the shoulder muscles to reinforce normal movement patterns. In their investigation, electromyography results showed a sequential pattern including deactivation of the left soleus, activation of the right tensor fasciae latae and rectus femoris, activation of the left semitendinosus and gluteus maximus, and, finally, activation of the right erector spinae before the initial deltoid activity. They concluded that weak core stabilizers led to distal segment injury that altered the proximal-to-distal control.

Tse et al. [43] argued that an 8-week core stability training program resulted in no significant changes in any performance tests among college-age rowers. Stanton et al. [34] also demonstrated that core stability exercises did not significantly improve physical performance in high school football and basketball athletes. Their results, which oppose those of the current study, may be due to many factors, including the different sport practised, the type and efficacy of the applied training program, and the program duration. Thus, they cannot decrease the important role of core training in providing proximal stability for distal mobility, minimizing the risk of shoulder injuries.

\section{Limitations}

The present study has some limitations. (a) The measurement of trunk isokinetic PT was performed in a sitting position, in accordance with the Biodex isokinetic dynamometer manual, which was not functional for swimming. (b) The results of this study may not be applicable to all swimmers with SSS as acromioclavicular and sternoclavicular joints pathology. (c) Participant withdrawal from the study was its another limiting factor. (d) The study lasted for 8 months, which means that some athletes were tested during the early preseason period (not reaching high performance), while others were investigated during the competitive season (maximal performance).

\section{Conclusions}

Musculoskeletal overuse injuries are the main source of shoulder pain in competitive swimmers. There is a vicious circle in the relationship between the weakness of core muscles and shoulder injury. Weak core inhibits shoulder muscles, which is a major risk factor for shoulder instability and SSS. The development of core muscle strength and durability decreases the reaction time at which these muscles are activated as a preparation for making corset-like posture before distal movement and this, in turn, minimizes shoulder injuries. Thus, it is very important to incorporate a core stability training program into the rehabilitation plan to provide proximal stability for obtaining safe and proper distal mobility across the shoulder joint.

\section{Acknowledgements}

The authors thank all participants in the study for their cooperation.

\section{Disclosure statement}

No author has any financial interest or received any financial benefit from this research.

\section{Conflict of interest}

The authors state no conflict of interest.

\section{References}

1. Wolf BR, Ebinger AE, Lawler MP, Britton CL. Injury patterns in Division I collegiate swimming. Am J Sports Med. 2009;37(10):2037-2042; doi: 10.1177/0363546509339 364.

2. O'Donnell CJ, Bowen J, Fossati J. Identifying and managing shoulder pain in competitive swimmers: how to minimize training flaws and other risks. Phys Sportsmed. 2005;33(9):27-35; doi: 10.3810/psm.2005.09.195.

3. Madsen PH, Bak K, Jensen S, Welter U. Training induces scapular dyskinesis in pain-free competitive swimmers: a reliability and observational study. Clin J Sport Med. 2011;21(2):109-113; doi: 10.1097/JSM.0b013e318204 $1 \mathrm{de} 0$.

4. Borsa PA, Laudner KG, Sauers EL. Mobility and stability adaptations in the shoulder of the overhead athlete: a theoretical and evidence-based perspective. Sports Med. 2008;38(1):17-36; doi: 10.2165/00007256-20083 8010-00003. 
5. Oyama S. Profiling physical characteristics of the swimmer's shoulder: comparison to baseball pitchers and nonoverhead athletes. Master's thesis. Pittsburgh: University of Pittsburgh; 2006.

6. Hibberd EE, Myers JB. Practice habits and attitudes and behaviors concerning shoulder pain in high school competitive club swimmers. Clin J Sport Med. 2013; 23(6):450-455; doi: 10.1097/JSM.0b013e31829aa8ff.

7. Sein ML, Walton J, Linklater J, Appleyard R, Kirkbride B, Kuah D, et al. Shoulder pain in elite swimmers: primarily due to swim-volume-induced supraspinatus tendinopathy. Br J Sports Med. 2010;44(2):105-113; doi: 10.1136/ bjsm.2008.047282.

8. De Martino I, Rodeo SA. The swimmer's shoulder: multidirectional instability. Curr Rev Musculoskelet Med. 2018; 11(2):167-171; doi: 10.1007/s12178-018-9485-0.

9. Hill L, Collins M, Posthumus M. Risk factors for shoulder pain and injury in swimmers: a critical systematic review. Phys Sportsmed. 2015;43(4):412-420; doi: 10.1080/00 913847.2015.1077097.

10. Nichols AW. Medical care of the aquatics athlete. Curr Sports Med Rep. 2015;14(5):389-396; doi: 10.1249/ JSR.0000000000000194.

11. Bak K, Faunø $P$. Clinical findings in competitive swimmers with shoulder pain. Am J Sports Med. 1997;25(2):254260; doi: 10.1177/036354659702500221.

12. Matzkin E, Suslavich K, Wes D. Swimmer's shoulder: painful shoulder in the competitive swimmer. J Am Acad Orthop Surg. 2016;24(8):527-536; doi: 10.5435/JAAOSD-15-00313.

13. Wanivenhaus F, Fox AJS, Chaudhury S, Rodeo SA. Epidemiology of injuries and prevention strategies in competitive swimmers. Sports Health. 2012;4(3):246-251; doi: 10.1177/1941738112442132.

14. Kibler WB, Ludewig PM, McClure PW, Michener LA, Bak K, Sciascia AD. Clinical implications of scapular dyskinesis in shoulder injury: the 2013 consensus statement from the 'Scapular Summit'. Br J Sports Med. 2013;47(14): 877-885; doi: 10.1136/bjsports-2013-092425.

15. Akuthota V, Ferreiro A, Moore T, Fredericson M. Core stability exercise principles. Curr Sports Med Rep. 2008; 7(1):39-44; doi:10.1097/01.CSMR.0000308663.13278.69.

16. Fredericson M, Moore T. Muscular balance, core stability, and injury prevention for middle- and long-distance runners. Phys Med Rehabil Clin N Am. 2005;16(3):669-689; doi: 10.1016/j.pmr.2005.03.001.

17. Zazulak BT, Hewett TE, Reeves NP, Goldberg B, Cholewicki J. The effects of core proprioception on knee injury: a prospective biomechanical-epidemiological study. Am J Sports Med. 2007;35(3):368-373; doi: 10.1177/ 0363546506297909.

18. Behm DG, Leonard AM, Young WB, Bonsey WAC, MacKinnon SN. Trunk muscle electromyographic activity with unstable and unilateral exercises. J Strength Cond Res. 2005;19(1):193-201; doi: 10.1519/1533-4287(2005) 19<193:TMEAWU>2.0.CO;2.

19. Sahrmann S. Diagnosis and treatment of movement impairment syndromes. St. Louis: Mosby; 2002.

20. Richardson CA, Snijders CJ, Hides JA, Damen L, Pas MS, Storm J. The relation between the transverse abdominis muscle, sacroiliac joint mechanics, and low back pain. Spine. 2002;27(4):399-405; doi: 10.1097/00007632200202150-00015.

21. Barr KP, Griggs M, Cadby T. Lumbar stabilization: core concepts and current literature, part 1. Am J Phys Med 163709.70471 .42 .
22. McGill S. Low back disorders: evidence-based prevention and rehabilitation. Champaign: Human Kinetics; 2002.

23. Matsen FA $3^{\text {rd }}$, Chebli C, Lippitt S, American Academy of Orthopaedic Surgeons. Principles for the evaluation and management of shoulder instability. J Bone Joint Surg Am. 2006;88(3):648-659; doi: 10.2106/00004623-2006 03000-00026.

24. Cools AM, Geerooms E, Van den Berghe DF, Cambier DC, Witvrouw EE. Isokinetic scapular muscle performance in young elite gymnasts. J Athl Train. 2007;42(4):458-463.

25. Turgut E, Duzgun I, Baltaci G. Effects of scapular stabilization exercise training on scapular kinematics, disability, and pain in subacromial impingement: a randomized controlled trial. Arch Phys Med Rehabil. 2017;98(10): 1915-1923.e3; doi: 10.1016/j.apmr.2017.05.023.

26. Beasley L, Faryniarz DA, Hannafin JA. Multidirectional instability of the shoulder in the female athlete. Clin Sports Med. 2000;19(2):331-349; doi: 10.1016/s02785919(05)70207-6.

27. Bertocci GE, Munin MC, Frost KL, Burdett R, Wassinger CA, Fitzgerald SG. Isokinetic performance after total hip replacement. Am J Phys Med Rehabil. 2004;83(1):19; doi: 10.1097/01.PHM.0000098047.26314.93.

28. Drouin JM, Valovich-McLeod TC, Shultz SJ, Gansneder BM, Perrin DH. Reliability and validity of the Biodex System 3 Pro isokinetic dynamometer velocity, torque and position measurements. Eur J Appl Physiol. 2004; 91(1):22-29; doi: 10.1007/s00421-003-0933-0.

29. Remaud A, Cornu C, Guével A. A methodologic approach for the comparison between dynamic contractions: influences on the neuromuscular system. J Athl Train. 2005;40(4):281-287.

30. Kongsgaard M, Reitelseder S, Pedersen TG, Holm L, Aagaard P, Kjaer M, et al. Region specific patellar tendon hypertrophy in humans following resistance training. Acta Physiol. 2007;191(2):111-121; doi: 10.1111/j.17481716.2007.01714.x.

31. Dvir Z, Keating J. Reproducibility and validity of a new test protocol for measuring isokinetic trunk extension strength. Clin Biomech. 2001;16(7):627-630; doi: 10.1016/s02680033(01)00038-9.

32. Aly SM, Abd El-Mohsen AM, El Hafez SM. Effect of six weeks of core stability exercises on trunk and hip muscles' strength in college students. Int $\mathrm{J}$ Ther Rehabil Res. 2017;6(2):9-15; doi: 10.5455/ijtrr.000000237.

33. Shirado O, Ito T, Kaneda K, Strax TE. Flexion-relaxation phenomenon in the back muscles. A comparative study between healthy subjects and patients with chronic low back pain. Am J Phys Med Rehabil. 1995;74(2):139-144.

34. Stanton R, Reaburn PR, Humphries B. The effect of shortterm Swiss ball training on core stability and running economy. J Strength Cond Res. 2004;18(3):522-528; doi: 10.1519/1533-4287(2004)18<522:TEOSSB>2.0.CO;2.

35. Willardson JM. Core stability training: applications to sports conditioning programs. J Strength Cond Res. 2007;21(3):979-985; doi: 10.1519/R-20255.1.

36. Zedka M, Prochazka A, Knight B, Gillard D, Gauthier M. Voluntary and reflex control of human back muscles during induced pain. J Physiol. 1999;520(2):591-604; doi: 10.1111/j.1469-7793.1999.00591.x.

37. Fritz JM, Cleland JA, Childs JD. Subgrouping patients with low back pain: evolution of a classification approach to physical therapy. J Orthop Sports Phys Ther. 2007;37(6): 290-302; doi: 10.2519/jospt.2007.2498.

38. Sinnott KA, Milburn P, McNaughton H. Factors associated with thoracic spinal cord injury, lesion level and ro- 
tator cuff disorders. Spinal Cord. 2000;38(12):748-753; doi: 10.1038/sj.sc.3101095.

39. Rizio L, Uribe JW. Overuse injuries of the upper extremity in baseball. Clin Sports Med. 2001;20(3):453-468; doi: 10.1016/s0278-5919(05)70262-3.

40. Neumann DA. Kinesiology of the musculoskeletal system: foundations for physical rehabilitation, $2^{\text {nd }}$ ed. St. Louis: Mosby; 2010.

41. Cholewicki J, Juluru K, McGill SM. Intra-abdominal pressure mechanism for stabilizing the lumbar spine. J Biomech. 1999;32(1):13-17; doi: 10.1016/s0021-9290(98) 00129-8.

42. Johnson JN, Gauvin J, Fredericson M. Swimming biomechanics and injury prevention: new stroke techniques and medical considerations. Phys Sportsmed. 2003;31(1): 41-46; doi: 10.3810/psm.2003.01.165.

43. Tse MA, McManus AM, Masters RSW. Development and validation of a core endurance intervention program: implications for performance in college-age rowers. $J$ Strength Cond Res. 2005;19(3):547-552; doi: 10.1519/ 15424.1. 